\title{
DRG-Begleitforschung: jetzt scharf stellen
}

\section{Michel Romanens}

* Der folgende Text widerspiegelt ausschliesslich die Meinung des Autors.

1 www.tagesanzeiger.ch/ schweiz/standard/Spitaelerlehnen-die-Weitergabevon-Patientendaten-ab/ story/13010131

Korrespondenz:

Dr. med. Michel Romanens

Innere Medizin und Kardiologie

FMH

Ziegelfeldstrasse 1

CH-4600 Olten

info@kardiolab.ch

\section{Einleitung}

Am 8. August 2011 hat der Verein Ethik und Medizin Schweiz (VEMS) zusammen mit der Interessengemeinschaft für das Kantonsspital Olten die Begleitforschung zu den Fallpauschalen diskutiert. Ein Einführungsreferat des VEMS sowie drei Fachvorträge von Vertretern/-innen der Pflegewissenschaft (Maria Schubert), der FMH (Beatrix Meyer) und des Nationalen Vereins für Qualitätsentwicklung in Spitälern und Kliniken ANQ (Thomas Straubhaar) bildeten die Grundlage für die Diskussion. Am Podium nahm anschliessend zudem Nationalrätin Bea Heim teil. Unser Beitrag soll die wichtigsten offenen Fragen und erste Lösungsansätze thematisieren. *

Mit der Einführung der Fallpauschalen 2012 wird das System scharf gestellt. Dass dies mit betriebswirtschaftlichen Problemen verbunden ist, zeigt die Weigerung von $81 \%$ der $\mathrm{H}+$ Verbandsspitäler, die Vereinbarungen zwischen $\mathrm{H}+$ und der santésuisse zu akzeptieren [1]. Doch auch andere Überlegungen sind angebracht: Die Mittel zur Durchführung der Vergütungsform nach DRG müssen nach KVG Art. 56 wirksam, zweckmässig und wirtschaftlich sein, ansonsten eine Bezahlung der Codierungen zumindest aus der Grundversicherung problematisch ist. Entsprechen nämlich diese Kosten für die Behandlung der Patientin nicht den WZW-Kriterien, erwartet die Spitäler, welche nach DRG abrechnen, gemäss Art. 59 KVG eine Strafe.

Es wurde offenbar zu wenig bedacht, dass mit der neuen dualen Spitalfinanzierung die Versicherungsprämien aus der Grundversicherung direkt die Spitäler zu $45 \%$ finanzieren sollen. Damit gilt der Grundsatz der WZW-Regeln selbstverständlich auch für die Kosten, welche durch die Berechnung der Rechnungen anfallen. Demnach müsste dem Gesetz folgend zunächst die WZW-Tauglichkeit der DRG nachgewiesen werden, bevor Prämiengelder verwendet werden dürfen. Da mittlerweile alle bezweifeln, dass die Spitalkosten dank DRG abnehmen, ist davon auszugehen, dass die DRGs die Vorgaben aus dem KVG Art. 56 nicht erfüllen. Da sogar damit zu rechnen ist, dass die Patientensicherheit mit dem DRG-System abnimmt, ist zeitgleich die Scharfstellung der Begleitforschung geboten. Doch wie soll dies geschehen?

\section{Mittelwert oder Audits}

Wir unterscheiden zwischen Vergleichsforschung (Mittelwertvergleiche) und Ursachenforschung (Audits). Die Vergleichsforschung generiert Mittelwerte, wobei beobachtete Unterschiede ohne vertiefte Forschung nicht erklärbar sind. Dies erhöht das Risiko,

\section{Comment focaliser la recherche} concomitante?

L'examen de la situation actuelle montre que la recherche concomitante est tout à fait en mesure d'objectiver les effets indésirables d'importance clinique des forfaits par cas, notamment à l'aide de I'indice BERNCA, qui effectue non seulement des comparaisons de moyennes, comme le propose I'Association nationale pour le développement de la qualité dans les hôpitaux et les cliniques (ANQ), mais aussi des recherches de causalité directe.

Nous devons exiger que l'indice BERNCA soit provisoirement défini comme le «gold standard» dans l'évaluation des conséquences des forfaits par cas pour la sécurité des patients. II convient par conséquent d'élargir son emploi, d'améliorer au besoin son financement et de poursuivre sa validation par des données de résultats.

Il faut faire en sorte que les instances politiques et le public se voient communiquer des résultats cliniquement pertinents et obtiennent, si nécessaire, la possibilité d'éliminer le système de forfaits par cas de la LAMal.

Une instance indépendante doit être créée qui puisse examiner tous les résultats à tout moment. Parce qu'elle doit être totalement séparée financièrement des acteurs du système de santé, cette instance doit être financée par une structure appropriée, p. ex. une fondation. Cette condition est la seule à même de garantir suffisamment l'indépendance de la science dans ces questions importantes que sont la sécurité des patients et la qualité des soins.

La FMH s'est déjà très fortement impliquée pour une conduite scrupuleuse de la recherche concomitante.

La Fédération, et avec elle bien sûr tout le corps médical, se doit de maintenir ses exigences d'une recherche concomitante aussi solidement validée que possible dans l'intérêt de la sécurité des patients. 
dass wissenschaftlich nicht zulässige Schlüsse aus solcher Forschung gezogen werden. Der Sinn der Mittelwertvergleiche liegt damit lediglich in der Generierung von Unterschieden, welche den Institutionen mitgeteilt werden. Dies ist die eigentliche Leistung des ANQ.

Es obliegt dann den beobachteten Institutionen, die eigentlichen Ursachen für Mittelwert-Unterschiede zu erforschen um festzustellen, ob überhaupt ein Problem vorliegt. Denn die meisten Unterschiede haben entgegen gängiger Meinung nichts mit der Performance einer Institution zu tun, sondern sind Ausdruck von Co-Variablen (statistisch relevante Faktoren, welche Beobachtungen erklären). Es wird zwar versucht, den Einfluss solcher Co-Variablen zu elimieren, indem zum Beispiel Morbiditätsvariablen in die Mittelwert-Vergleiche einfliessen. Leider ist diese und ist ein Beleg für die Problematik von Sterberaten als Performance-Kriterium.

Eine Routinekontrolle sämtlicher Sterbefälle bei insgesamt 7,6 Mio. Hospitalisationen zeigte in England, dass die Sterberaten höher waren in Regionen mit weniger Spitälern, weniger Ärzten und bei kränkeren Personen. Eine Senkung der Spitaldichte kann deshalb die Spitalmortalität erhöhen. Die Spitaldichte wird aber selten als Co-Variable in der korrigierten Spitalsterberate verwendet [3].

Betreffend publizierte Sterberaten des Bundesamtes für Gesundheit: Eine Nachkontrolle der Kardiologie des UniversitätsSpitals Zürich zeigte, dass das Bundesamt für Gesundheit (BAG) bezogen auf dieses Spital und Departement nicht korrekte Sterberaten für Koronareingriffe publizierte (sogenannter Type I Error mit «Data source and coding inaccuracies») [4].

\section{Die meisten Unterschiede haben entgegen gängiger Meinung nichts mit der Performance einer Institution zu tun, sondern sind Ausdruck von Co-Variablen.}

Forschung in den Resultaten nicht konsistent: Häufig treten trotz scheinbar adäquaten Korrekturen unwahrscheinliche Resultate auf.

Die Gefahr dieser Forschung liegt in der Überbewertung der Resultate. Einerseits können Institutionen Opfer eines «Mislabeling» werden, andererseits können Mittelwert-Unterschiede, falls veröffentlicht, leicht als Performance-Unterschiede fehlinterpretiert werden. Intrainstitutionelle oder unabhängige AuditVerfahren stellen demgegenüber eine zwar viel teurere, aber auf die eigentliche Ursachenforschung fokussierte Forschungsaktivität dar, welche betreffend Ursachen von Performance-Problemen (z. B. un-
Ein zentrales Problem der Sterberaten ist der sogenannte «Risk avoidance creep» [5]. Es wurde beispielsweise beobachtet, dass die veröffentlichen Sterberaten zu einem Vermeidungsverhalten bei der Behandlung von Koronareingriffen mit sehr hohem Sterberisiko (z. B. PTCA bei Herzinfarkt mit kardiogenem Schock) führen. Dies ist paradox, denn gerade solche Patienten können am meisten von diesem Noteingriff profitieren. Anstelle der Senkung des Zählers in den Sterberaten kann natürlich durch eine Ausweitung von Eingriffen mit sehr niedrigem Risiko der Nenner erhöht werden, womit die Sterberate ebenfalls sinkt. (ning mortality Hospital Standardized Mortality Rate between 2003 and 2005. BMC Health Services Research. 2008;8:73.

3 Jarman B, Gault S, Alves B et al. Explaining differences in English hospital death rates using routinely collected data. BMJ. 1999;318:1515-1520.

4 Lüscher T. Lies, Damn Lies, and Statistics. Bemerkungen zu den Outcome-Zahlen des Bundesamtes für Gesundheit. Kardiovaskuläre Medizin. 2009;12(9): 229-233 20.

5 Resnic FS, Welt FGP. The Public Health Hazards of Risk Avoidance Associated With Public Reporting of Risk-Adjusted Outcomes in Coronary Intervention. $\mathrm{J}$ Am Coll Cardiol. $2009 ; 53: 825-30$

\section{Es gibt Hinweise dafür, dass die zunehmende Forschung in den Spitälern zu übermässiger Personalbelastung führt.}

erwarteter Todesfall während der Spitalbehandlung) sämtliche Aspekte zu ergründen versucht. Damit ist die Audit-Forschung viel mehr der Wahrheit verpflichtet. Dies ist anhand der folgenden Diskussion zu den Sterberaten exemplifiziert.

\section{Beispiel Sterberaten}

Sterberaten im Spital sind seit Jahrzehnten Gegenstand intensiver Forschung weltweit. Eine holländische nationale Beobachtung korrigierter Sterberaten für die Jahre 2003-2005 unter Einschluss aller Spitäler zeigte die höchsten Sterberaten in den Universitätskliniken [2]. Diese Studie belegt, dass die Korrekturfaktoren für die Sterblichkeit ungenügend waren,
Solche künstlichen Eingriffe in Sterberaten sind tatsächlich eine Gefahr für die Patienten und mit den gesetzlichen Rahmenbedingungen des Medizinalberufegesetzes nicht vereinbar. Sterberaten unterliegen einer Vielzahl von Biasproblemen, neben dem «Data source und coding»- Problem sind eine Vielzahl von Daten kurz vor, während und nach der Hospitalisation Quelle von fehlerhafter Beurteilung der Performance von Institutionen.

Eine weiterführende Diskussion der Problematik von Sterberaten findet sich auf der Webseite des VEMS, insbesondere die beiden Beiträge «Inter-Institutional Profiling and Patient Safety: Which Method to Appropriately Assess the Future Risk of Flat Rates 
in Hospital Care?» [6] und «Swiss Flat Rates and Monitoring of Coronary Hospital Performances on Behalf of Serial Sentinel, Census, and Appropriateness Measurements» [7].

\section{Forschung und Personalbelastung}

Demnächst wird der ANQ einen Tageszensus zur Erfassung der Prävalenz von Dekubitalgeschwüren durchführen. Gemäss Pflegedienst der Solothurner Spitäler AG sind 46 Personen während eines Tages damit beschäftigt, diese Daten zu erheben. Es gibt Hinweise dafür, dass die zunehmende Forschung in den Spitälern zu übermässiger Personalbelastung führt mit der Konsequenz, dass die Studienblätter entweder gar nicht oder mangelhaft ausgefüllt werden. Dies ist ein potentiell wesentlicher Bias in der Begleitforschung. Es ist bisher nicht klar, wie dieser Bias erfasst wird.

\section{Abgleich und Validierung von Datenbanken}

Die ursprünglich von der FMH in ihrem Grundsatzpapier vorgesehene koordinatorische Aufgabe der Aktiengesellschaft Swiss DRG kam nicht zustande. Wir haben jedoch bereits über den Interessenkonflikt in diesem Konzept publiziert und sind nicht unglücklich darüber [8]. Die Gefahr der Partikularisierung der Datenerhebungen ist jedoch programmiert. Durch fehlende Koordination und Abgleich besteht die Möglichkeit (oder Gefahr?), dass in zwei oder mehreren Forschungsprojekten beispielsweise Komplika-
Gerade beim ethischen Konflikt zwischen dem behandelnden Arzt und der Institution (Spital, Managed Care) können Anreize geschaffen werden, die Gewinne erlauben, welche nicht vollumfänglich dem Interesse der Patienten dienen. Wie solche Effekte erfasst werden, scheint bisher noch ungenügend erforscht. Zudem muss zunächst auch rechtswissenschaftlich ausgeleuchtet werden, ob die gesetzlichen Vorgaben zu Fallpauschalen und Budgetmitverantwortung mit dem MedBG und dem OR überhaupt je kompatibel sind. Gemäss einem Rechtsgutachten der Unifversität St.Gallen sind juristische Bedenken in diesem Kontext jedenfalls angebracht [9].

\section{Kostendeckungsforschung}

Gemäss gesetzlichen Vorgaben im KVG müssen die Fallpauschalen kostendeckend sein. Obwohl eine Kostenneutralität sinngemäss wie im TARMED nicht vorgesehen ist, schreibt Art. 59c KVV vor, dass der Wechsel zum System der Fallpauschalen nicht zu Mehrkosten führen darf [10]. Die Kostendeckung gemäss Regressionsanalysen der SwissDRG betragen rund $48 \%$ ohne Inlyers und ohne Outlyers, mit diesen $78 \%$ [11]. Das heisst, es droht Unterdeckung der Kosten. Die Kostendeckung anhand von Regressionsanalysen kann je nach Fachgebiet erheblich variieren und ist besonders in der Pädiatrie notorisch tief.

So weit bekannt ist, existieren keine Pläne für die Durchführung einer Kostendeckungsforschung. Es muss aber an dieser Stelle betont werden, dass Spital-
6 www.physicianprofiling.ch/ VEMSFlatrates2010.pdf

7 www.physicianprofiling.ch/ VEMSAudits2010.pdf

8 www.saez.ch/pdf_d/ 2010/2010-07/2010-071036.pdf

9 Gutachten zuhanden des Vereins Ethik und Medizin Schweiz zu Fragen des Managed-Care-Systems von PD Dr. iur. Ueli Kieser, Vizedirektor IPR-HSG, Rechtsanwalt, Zürich / St. Gallen. August 2011.

10 www.santesuisse.ch/de/ dyn_output.html?content. cdid $=29527 \&$ content. vcid $=6 \&$ detail $=$ yes

11 www.physicianprofiling.ch/ SwissDRGRisikoausgleich2007.pdf

12 www.physicianprofiling.ch/ PHYPublicHealthStellungsnahmeCassis.pdf

13 www.dgepi.de/doc/ Empfehlungen.doc

\section{Es ist sicherzustellen, dass Politik und Öffentlichkeit klinisch relevante Resultate erhalten und falls nötig die Möglichkeit haben, das Fallpauschalen-System aus dem KVG zu eliminieren.}

tionsraten, wie nosokomiale Infektionen in gleicher oder in unterschiedlicher Art und Weise erfasst werden, ohne dass hierüber kommuniziert wird oder die Resultate miteinander verglichen werden. Ist es sinnvoll, dass diese Probleme doppelt gemessen werden, und falls ja, warum ist nicht vorgesehen, diese Resultate zu vergleichen?

\section{Rechtliche Rahmenbedingungen und Gewinne}

Gemäss Obligationenrecht Art. 39 ff und Medizinalberufegesetz Art. 40 ff. existieren klar definierte rechtliche Rahmenbedingungen für die ärztliche Tätigkeit. Ziel dieser Gesetzgebung ist die Vermeidung von Korruption, welche gesundheitspolizeilich geahndet werden kann. Das Medizinalberufegesetz steht zudem über dem Sozialversicherungsrecht (z. B. Krankenversicherungsgesetz) und verbietet ärztliche Tätigkeiten in der Arzt-Patienten-Beziehung, welche nicht ausschliesslich den Interessen des den Behandlungsauftrag erteilenden Patienten dienen. schliessungen wegen ungenügender Kostendeckung erfolgen könnten. Diese Forschung ist deswegen als zentral zu betrachten.

\section{Qualität der Begleitforschungsinstrumente}

Es ist unabdingbar, dass jede Schlussfolgerung aus einer Studie betreffend den «Level of evidence» charakterisiert wird [12]. Kommunikation des «Levels of evidence» in der Presse sollte sich zudem strikt an diese Vorgaben halten. Betreffend den Umgang mit «Confounding variables» sollten zudem die Richtlinien der «Good epidemiological practice» berücksichtigt werden [13].

\section{Resultate und Geheimhaltung}

Die Forschungsaktivitäten in diesem Bereich bewegen sich aufgrund ihrer Bedeutung sowie der Erhebung der Daten in öffentlichen Spitälern im öffentlich-rechtlichen Raum und generieren ein hohes öffentliches Interesse, zumal es um die Patientensicherheit geht. Ob eine Datenerhebung in Privatspi- 
tälern den gleichen Prämissen unterliegt, scheint kaum bekannt. In jedem Fall ist zu fordern, dass interessierte Personen die - anonymisierten - Datensätze einsehen und analysieren können. Zudem sind die Vorgaben der Charta des Ethikrates für Statistik zu berücksichtigen [14].

\section{Resultate und Konsequenzen auf kantonaler Ebene}

Es ist denkbar, dass künftige Forschung zeigen wird, dass wegen Personalabbau und Leistungsdruck die Patientensicherheit im Spital abnimmt bzw. die Komplikationsraten zunehmen. Dies kann bereits heute mit einem validierten Instrument beobachtet werden (BERNCA-Index), welcher gemäss Auffassung des VEMS eine Mischung darstellt zwischen MittelwertBeobachtung und im Fragebogen erfassten Variablen, ähnlich einem Audit. Wenn nun in einem Spital ent- relevante Nebenwirkungen der Fallpauschalen zu objektivieren, vor allem in Form des BERNCA-Indexes, welcher nicht nur Mittelwert-Vergleiche durchführt wie vom ANQ vorgeschlagen, sondern auch direkte Ursachenforschung betreibt.

Es ist zu fordern, dass der BERNCA-Index als vorläufiger Goldstandard in der Beurteilung der Auswirkungen von Fallpauschalen auf die Patientensicherheit definiert wird. Er ist demnach auch breit einzusetzen, allenfalls noch besser $\mathrm{zu}$ finanzieren und weiter mit Outcome-Daten zu validieren.

Es ist sicherzustellen, dass Politik und die Öffentlichkeit klinisch relevante Resultate erhalten und falls nötig die Möglichkeit haben, das Fallpauschalen-System aus dem KVG zu eliminieren.

Wichtige Aspekte der Begleitforschung, insbesondere legaler, qualitativer und ethischer Art, sind noch besser zu definieren und zu finanzieren.

\section{Es ist weiterhin Sache der FMH, eine möglichst gut validierte Begleit- forschungsaktivität im Interesse der Patientensicherheit einzufordern.}

sprechend auffällige Feststellungen gemacht werden, muss sichergestellt werden, dass geeignete Gegenmassnahmen umgehend eingeleitet werden. Die Frage ist, wer solche Probleme überwacht. Die öffentlichen Spitäler sind selbst in der Form der Aktiengesellschaft vollumfänglich im Besitz der Kantone. Das Kantonsparlament muss somit über den politischen Weg die Transparenz einfordern. Wie dieses Problem für private Spitäler zu lösen ist, ist unklar.

\section{Resultate und künftige Gesundheitspolitik auf nationaler Ebene}

Transparent gemachte, öffentlich zugängliche und validierte Resultate der Begleitforschung müssen in den Fällen Konsequenzen haben, in denen nachweisbar ist, dass Fallpauschalen zu einer Abnahme der Patientensicherheit oder zu einer Mengenausweitung medizinischer Eingriffe führen, allenfalls auch mit dem Medizinalberufegesetz oder dem Obligationenrecht nicht kompatibel sind oder erhebliche ethische Konflikte bei den Leistungserbringern auslösen. Es muss möglich sein, einen öffentlichen und politischen Diskurs darüber zu führen, ob die Fallpauschalen bei überbordenden negativen Auswirkungen aus dem Krankenversicherungsgesetz als Abgeltungssystem eliminiert werden.

www.stat.ch/docs/ethics/ charter-g.pdf

15 www.physicianprofiling.ch/VEMSBegleitforschungV0.1.pdf
Wie stellen wir die Begleitforschung scharf? Die Bestandsaufnahme August 2011 zeigt, dass die Begleitforschung durchaus in der Lage ist, klinisch
Es sollte eine unabhängige Stelle geschaffen werden, welche alle Resultate jederzeit einsehen kann. Diese Stelle muss finanziell von den Playern im Gesundheitswesen vollkommen entkoppelt werden und ist entsprechend zu finanzieren, z.B. über eine Stiftung. Damit wird die Unabhängigkeit der Wissenschaft in diesen wichtigen Fragen der Patientensicherheit und Versorgungsqualität erst ausreichend gewährleistet.

Die FMH hat sich bereits intensiv für die korrekte Durchführung der Begleitforschung eingesetzt. Es ist weiterhin Sache der FMH und mit ihr selbstverständlich auch der Ärzteschaft, eine möglichst gut validierte Begleitforschungsaktivität im Interesse der Patientensicherheit einzufordern. Der VEMS hat dazu die Begründungen und den Forderungskatalog festgeschrieben [15].

Die Doktrin «Entscheid ist letztlich Sache der Politik» blendet sowohl die rechtlichen als auch die standesethischen Richtlinien aus - damit machen wir es uns zu einfach. Es ist genuine Sache der Ärzteschaft selbst - und basierend auf demokratischen Ärzte-Hearings dann Sache der FMH -, auf höchstem professionellem (im wahren Sinn dieses Wortes) Niveau Begleitforschung zu definieren und dann einzufordern und die Leitplanken, innerhalb derer die Führung des Gesundheitswesens stattzufinden hat, unmissverständlich und eindeutig zu kommunizieren. 